\title{
Uma Análise da Confiança na Qualidade do Contexto em VANETs
}

\author{
Margarete Oliveira dos S. de Sá e Sérgio Gorender \\ Laboratório de Sistemas Distribuídos (LaSiD) \\ Programa de Pós-Graduação em Mecatrônica (PPGM) \\ Departamento de Computação Interdisciplinar (DCI) \\ Instituto de Computação (IC) - Universidade Federal da Bahia (UFBA) \\ Corresponding author: (magsa, gorender)@ufba.br.
}

\begin{abstract}
Resumo-Em sistemas cientes do contexto (Context-aware systems - CAS), informações sobre o ambiente obtidas de sensores são utilizadas pelo sistema para se adaptar de forma autônoma às alterações deste ambiente. Métricas de Qualidade de Contexto (QoC) apoiam a decisão destes sistemas quanto ao uso do contexto. Em VANETs o contexto pode estar relacionado com informações da mobilidade dos veículos ou de seu ambiente de comunicação, mas também das aplicações sendo executadas e seus usuários, assim como o conteúdo das mensagens sendo trocadas. Neste artigo utilizamos um contexto de mobilidade e comunicação para VANETs e consideramos as métricas de Qualidade de Contexto validity Time, Minimum Validity Time, Age e Confidence. Analisamos de forma detalhada o comportamento da métrica Confidence, a qual indica um nível de confiança para a métrica Validity Time, caracterizando QoC em níveis. Discutimos também, os resultados de implementação em um ambiente simulado (VEINS/SUMO/OMNET++).

Abstract-In context-aware systems (CAS), information about the environment obtained from sensors is used by the system to adapt itself autonomously to changes in this environment. Quality of Context (QoC) metrics support the decision of these systems regarding the use of context information. In VANETs, the context can be related to information about the mobility of vehicles or their communication environment, but also about the applications being executed and their users, as well as the content of the messages being exchanged. In this article we use a mobility and communication context for VANETs and consider the Quality of Context metrics validity Time, Minimum Validity Time, Age and Confidence. We analyze in detail the behavior of the Confidence metric, which indicates a confidence level for the Validity Time metric, characterizing QoC in levels. We also discuss the implementation results in a simulated environment (VEINS/SUMO/OMNET++).

Index Terms-VANET, Context, Quality of Context
\end{abstract}

\section{INTRODUÇÃO}

Em sistemas cientes do contexto (Context-Aware Systems $C A S$ ) informações coletadas do ambiente são utilizadas pelo sistema para tomar decisões relativas à sua execução, adaptando seu comportamento a alterações em diferentes aspectos deste ambiente. $\mathrm{O}$ conceito de sistemas cientes do contexto foi inicialmente proposto para sistemas pervasivos e sistemas baseados em sensores, nos anos 90, sendo que o termo contextaware systems foi apresentado por Schilit and Theimer [1] descrevendo diferentes aspectos e tipos de contexto e seu uso

O presente trabalho foi realizado com apoio da Fundação de Amparo à Pesquisa do Estado da Bahia (FAPESB) - Brasil. por sistemas. Pode-se considerar que Contexto (Context) é qualquer informação que pode ser usada para caracterizar a situação de entidades ditas relevantes para os sistemas e seus ambientes de execução, incluindo informações dos próprios usuários e das aplicações. Assim, os sistemas cientes do contexto são aqueles que utilizam o contexto para adaptar suas operações de forma transparente, aumentando a usabilidade e a eficácia do sistema [2], [3], [4].

$\mathrm{O}$ conceito de contexto tem sido utilizado em redes veiculares ad hoc (VANET - Vehicular Ad Hoc Network) com diferentes objetivos [5], [6], [7], [8]. Nestas redes, o contexto pode ser relativo a aspectos da mobilidade dos veículos, da comunicação, das aplicações executadas ou dos próprios usuários e motoristas. Estas informações podem auxiliar tanto o próprio sistema da rede, quanto as aplicações veiculares sendo executadas.

Para que as informações de contexto sejam utilizadas de forma adequada, foram propostas métricas para avaliar e qualificar estas informações, caracterizando o conceito de Qualidade de Contexto (Quality of Context - QoC) [9]. Qualidade de Contexto foi também proposto inicialmente para sistemas baseados em sensores. Nestes sistemas, métricas foram propostas baseadas em diferentes aspectos do funcionamento dos sensores e também nos requisitos de aplicações, relativos ao funcionamento do sistema. As métricas de QoC avaliam o quanto uma informação de contexto é acurada, recente e confiável, ajudando as aplicações a tomarem decisões adequadas [10], [11], [12], [13], [9].

Um contexto de mobilidade e comunicação para redes veiculares, conjuntamente com algumas métricas de Qualidade de Contexto foram apresentados anteriormente por Sá e Gorender [14]. O contexto é coletado por um sistema de monitoração de contexto [15], o qual acompanha aspectos da comunicação e da mobilidade dos veículos que se comunicam. São utilizadas na construção deste contexto informações de posicionamento e mobilidade dos veículos, e informações relativas a características dos sinais de comunicação sem fio destes veículos. Estas informações são obtidas de sensores como GPS (Global Position System) e acelerômetro, e dos dispositivos de comunicação, e são trocadas entre os veículos através do envio de mensagens pela rede.

As métricas de QoC propostas são calculadas a partir das 
informações do contexto. Foram propostas as métricas Validity Time, Minimum Validity Time, Timeliness, Age e Confidence [14].

A métrica de QoC Validity Time [14] foi apresentada em termos de seu comportamento, no contexto de um par de veículos que se comunicam. Mesmo com resultados iniciais de experimentos em ambiente simulado, verificou-se a possibilidade de utilizar o intervalo estimado de tempo de comunicação entre dois veículos para definir o prazo de validade da informação do contexto de comunicação entre dois veículos comunicáveis em VANETs [14].

Devido à dinâmica do ambiente de comunicação veicular as informações do contexto podem perder sua qualidade não sendo então adequadas para uso pelos sistemas. Questões como por exemplo, a autonomia da mobilidade dos veículos, a heterogeneidade dos sensores de mobilidade, as características das edificações nas vias, a falta de garantias da comunicação sem fio etc., podem alterar o estado das VANETs e suas aplicações. A métrica de QoC Confidence [14] conceitua o valor de Validity Time definindo um grau de confiança, baseado nas possíveis situações do ambiente. Esta confiança pode apoiar a tomada de decisão dos usuários do contexto quanto à forma de uso das informações contidas nesse contexto. Estes usuários podem ser sistemas de roteamento, mecanismos de disseminação de dados, diversas aplicações veiculares, entre outros.

Neste artigo analisamos com detalhes o conceito da métrica de Qualidade de Contexto Confidence. Também caracterizamos Confidence como uma métrica que qualifica uma outra métrica de QoC, a Validity Time. Além disto, apresentamos uma análise do comportamento da métrica Confidence em cenários com e sem perda de mensagens, variando os períodos entre envios de mensagens de contexto. Utilizamos o Framework Veins [16], [17] para a implementação e a simulação do sistema proposto.

O artigo está organizado da seguinte forma: a seção II descreve os conceitos sendo utilizados neste artigo, sendo composta pelas subseções: II-A define o modelo de sistema adotado; II-B descreve o contexto para comunicação veicular; e II-C descreve as métricas de QoC para VANETs utilizadas neste artigo; a seção seguinte, III, descreve a métrica Confidence e desenvolve uma discussão sobre esta métrica; a seção IV descreve a implementação desenvolvida em ambiente simulado, com os resultados apresentados na subseção IV-A; a seção V apresenta os trabalhos relacionados e a seção VI apresenta as conclusões deste artigo.

\section{Conceitos Fundamentais}

Nesta Seção, apresentamos os conceitos essenciais para o entendimento da discussão proposta neste artigo. Assim, apresentamos o modelo de sistema adotado, as informações do contexto envolvidas nesta análise e a descrição das métricas de QoC apresentadas em [14] e que serão utilizadas neste artigo.

\section{A. Modelo de Sistema}

Assumimos uma rede veicular ad-hoc (VANET), formada por veículos autônomos. Os veículos se comunicam por troca de mensagens via comunicação sem fio. A comunicação é assíncrona e não confiável. Mensagens podem ser perdidas ou entregues de forma incompleta.

Os veículos possuem capacidade para processamento e armazenamento de dados além de dispositivos para comunicação sem fio. Os veículos também possuem sensores tais como acelerômetro, velocímetro e GPS (Global Positioning System). Usando estes sensores os veículos consultam sua posição atual, assim como sentido, direção e velocidade de sua movimentação. O GPS também fornece a hora global atualizada, permitindo que os relógios dos veículos estejam sincronizados. Não existem restrições de energia nos veículos, uma vez que o funcionamento do motor mantém as baterias sempre carregadas.

Nas próximas seções apresentamos as informações do contexto e as métricas de QoC envolvidas da análise proposta.

\section{B. Contexto de Comunicação e Mobilidade para VANETs}

O contexto é construído utilizando as informações coletadas de sensores relativas às últimas posições do veículo, e sentido, direção e velocidade de sua movimentação [14]. A partir destas informações, o contexto também determina o estado de outros veículos, relativo à possibilidade de comunicação com o veículo local. Para isto, os veículos trocam suas informações de contexto, inserindo estas informações em mensagens das aplicações, ou utilizando mensagens especificas do ambiente de monitoração.

As possibilidades de comunicação são expressas em termos de um estado de vizinhança, e de um estado de comunicação. O contexto do estado de vizinhança, para cada veículo da rede, apresenta os seguintes valores com relação a cada outro veículo da rede:

- Neighbors - um veículo é um vizinho (neighbor) se sua distância para o veículo local é menor do que o alcance de seus sinais de comunicação sem fio $\left(\right.$ radius $\left._{r}\right)$;

- Non-Neighbors - a distância entre os veículos é maior do que o alcance de seus sinais de comunicação sem fio.

O contexto do estado de comunicação assume os seguintes valores:

- Communicating - um veículo remoto está em comunicação com o veículo local se são vizinhos, ou se existe uma rota de comunicação, através da VANET, entre os dois veículos;

- Non-Communicating - o veículo remoto não pode se comunicar com o veículo local.

Este contexto de comunicação pode ser utilizado pela VANET na construção de rotas de comunicação eficientes, e também pelas aplicações veiculares. Para cada informação de contexto coletada, também é obtido o seu momento (timestamp), o qual é utilizado no cálculo de métricas de Qualidade de Contexto.

\section{Qualidade do Contexto de comunicação e mobilidade em VANETs}

A métrica de Qualidade de Contexto Validity Time para o contexto de comunicação descrito na seção anterior representa 
o tempo estimado, durante o qual existe uma probabilidade de um veículo remoto se manter no estado Communicating com relação ao veículo local, considerando o contexto de mobilidade atual dos veículos [14]. Para veículos vizinhos (Neighbors), a métrica relaciona a velocidade relativa entre os veículos, suas últimas posições, e o alcance de seus sinais de comunicação sem fio, e calcula o tempo que os veículos levariam para sair de seus alcances de comunicação, mantidas suas movimentações atuais. O cálculo é dado por $V T_{r l}=\frac{S_{r l}}{r v_{r l}}$. $S_{r l}$ é a distância que falta para o veículo local sair do alcance da comunicação do veículo remoto, mantidas as atuais condições de movimentação dos dois veículos. Este cálculo é obtido a partir do alcance do sinal de comunicação do veículo remoto e a distância entre os veículos. $r v_{r l}$ é a velocidade relativa entre ambos, com base nos valores coletados mais recentemente.

Para veículos que não são vizinhos (non-neighbors), a existência de uma rota de comunicação entre ambos permite que os veículos estejam se comunicando entre si. Neste caso, é selecionado o menor valor de Validity Time entre os valores calculados para cada dois veículos vizinhos que compõem a rota.

Foram também propostas as métricas a seguir, como forma de qualificar este contexto [14]:

- Minimum Validity Time - é um valor de Validity Time, assumindo que os veículos se afastam à velocidade relativa máxima, percorrendo a menor distância possível para o final do alcance de comunicação, caracterizando uma situação de pior caso com relação às possíveis mudanças do contexto Communicating e Neighbors;

- Age - maior tempo decorrido relativo à última atualização das informações do contexto, caracterizando a idade deste contexto; para cada conjunto de informações de contexto, obtido de um sensor ou de outros veículos via mensagens, é considerado o momento (Timestamp) no qual estas informações foram atualizadas nos sensores; para a idade do contexto Communicating, é utilizado o Timestamp mais antigo entre as informações envolvidas na definição do respectivo estado de comunicação;

- Timeliness - o quão recente é o cálculo do Validity Time relativo ao próprio tempo de validade calculado; e

- Confidence - indica o grau de confiança definido para o valor calculado para o Validity Time.

Na próxima seção vamos analisar em detalhe o significado e comportamento da métrica Confidence, e na seção seguinte apresentaremos e discutiremos resultados obtidos por uma implementação do contexto e métricas em ambiente simulado.

\section{UMA ANÁLISE DA MÉTRICA Confidence DE QuAlidade DE CONTEXTO}

O Validity Time utiliza informações disponíveis no contexto de comunicação veicular que foram atualizadas de diferentes sensores, em instantes de tempos distintos. Além disso, varia de acordo com o tempo, com base nas informações mais recentemente atualizadas no contexto. Devido a essa dinâmica do ambiente, podem ocorrer mudanças não representadas ainda pelas atuais informações coletadas. Para lidar com estes casos, desenvolvemos a métrica de Qualidade do Contexto Confidence, que indica o grau de confiabilidade do valor calculado para o Validity Time, qualificando esta última.

Para isso, a Confidence classifica a idade (Age) com relação aos valores calculados para as métricas Validity Time e Minimum Validity Time, conforme descritas na seção II-C. O valor de Confidence é obtido relacionando a idade do contexto com o próprio validity Time, como uma indicação da probabilidade de a situação do ambiente de comunicação veicular ter sido modificada com relação ao que está representado no conteúdo do contexto, e em consequência, nos valores calculados para as métricas de QoC. A fórmula para o cálculo de Confidence está na Equação 1. Nesta equação, age $e_{r l}$ indica a métrica Age (idade) relativa ao contexto Communicating, considerando o estado de comunicação entre o veículo remoto e o veículo local. $V T_{r l}$ representa o Validity Time para o mesmo contexto Communicating e $V T_{r l}^{m i n}$ o Minimum Validity Time. $\alpha_{r l}$ representa o valor da métrica Confidence. Em todas as métricas, $r$ é o veículo remoto e $l$ o local, representando os veículos envolvidos no contexto de comunicação.

$$
\alpha_{r l}= \begin{cases}1-\frac{\left(a g e_{r l}-V T_{r l}^{\text {min }}\right)}{\left(V T_{r l}-V T_{r l}^{m i n}\right)} & : \text { if }\left(V T_{r l}^{\text {min }} \leq a g e_{r l}<V T_{r l}\right) \\ 1 & : \text { if }\left(a g e_{r l}<V T_{r l}^{\text {min }}\right) \\ 0 & : \text { otherwise }\end{cases}
$$

$\mathrm{Na}$ Equação 1, o valor de $\alpha_{r l}$ varia entre 0 e 1 , sendo que 1 indica que $V T_{r l}$ é confiável, e 0 indica que não se deve confiar no valor desta métrica e do contexto como um todo. A Figura 1 apresenta um exemplo do comportamento do valor de Confidence $\left(\alpha_{r l}\right)$ em relação à idade $\left(a g e_{r l}\right)$. Nesta Figura, enquanto age $r$ for menor que $V T_{r l}^{m i n}$, temos que $\alpha_{r l}$ é igual a 1 . Contudo, enquanto o valor de $a g e_{r l}$ for aumentando, $\alpha_{r l}$ vai sendo atenuado até que seu valor se torne 0 , quando age $r l \geq V T_{r l}$. No caso de uso de rotas para o encaminhamento de mensagens, Confidence será o menor valor calculado considerando as etapas da rota.

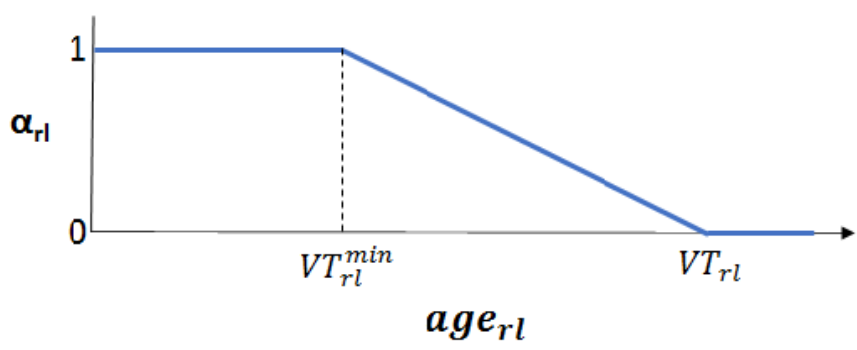

Figura 1: Um exemplo do comportamento de $\alpha_{r l}$ em relação a $a g e_{r l}$

O cálculo de Confidence compara o valor de Age (age $r$ ) com os valores de Validity Time $\left(V T_{r l}\right)$ e de Minimum Validity Time $\left(V T_{r l}^{\min }\right)$. Se $a g e_{r l}$ for menor que $V T_{r l}^{\min }$, temos que $\alpha_{r l}=1$, representando uma confiança de $100 \%$ no valor de Validity Time, como mostra a Figura 1. Neste caso, a idade 
do contexto Communicating é inferior ao Minimum Validity Time, indicando que, se não ocorreram falhas ou obstáculos à comunicação, o contexto Communicating continua válido representando o estado de comunicação entre um veículo remoto com relação ao veículo local, ou seja, os veículos não teriam se afastado e saído do alcance de seus sinais de comunicação sem fio. Com Confidence igual a 1, assumimos que o período calculado para o Validity Time se mantém como uma boa estimativa de tempo para a janela de comunicação dos veículos.

Por outro lado, caso age $e_{r l}$ seja maior que $V T_{r l}$, o período estimado em Validity Time terminou e o contexto não foi atualizado. Nesta situação, não é possível ter qualquer grau de confiança no valor calculado para $V T_{r l}$, e em consequência, na informação do contexto de comunicação entre os veículos. Neste caso, tanto é possível que apenas o tempo estimado tenha terminado e os veículos não mais se comuniquem, quanto o contexto esteja desatualizado e não mais represente a situação do ambiente de comunicação entre os dois veículos envolvidos.

Quando o valor de Age está entre o $V T_{r l}^{\min }$ e o $V T_{r l}$, a idade do contexto Communicating é inferior ao período calculado em Validity Time, embora maior que o Minimum Validity Time. O cálculo da confiança indicará que, quanto mais próxima a idade estiver de Minimum Validity Time, maior será o valor de Confidence, chegando próximo a $100 \%$, e quanto mais próxima esta idade estiver de Validity Time mais próximo de $0 \%$ estará o valor da confiança. À medida em que o tempo passa sem atualização do contexto Communicating, e a idade se aproxima do limite definido pelo $V T_{r l}$, o valor de Confidence é reduzido, indicando uma menor confiança no período do Validity Time.

O valor de Confidence pode ser utilizado para definir quando será necessário efetuar uma atualização do contexto, sendo que este momento pode ser relacionado, por exemplo, com os requisitos de uma aplicação, ou da própria rede. $\mathrm{O}$ valor de Confidence pode ser utilizado como um parâmetro para o sistema de monitoração de contexto da VANET, para definir prazos para a atualização de informações de contexto, tanto solicitando informações aos sensores locais, como enviando mensagens de solicitação de informações ao veículo remoto. Desta forma, tais mensagens de monitoração podem ser enviadas apenas quando necessárias, evitando aumentar a sobrecarga da rede. Por exemplo, uma aplicação pode definir $50 \%$ como o limite mínimo aceitável para Confidence, e sempre que este limite for alcançado, provocar o sistema de monitoração para atualizar o contexto, e em consequência, as métricas de QoC.

O comportamento da métrica Confidence qualifica o valor da métrica de QoC Validity Time do contexto Communicating, caracterizando um segundo nível de Qualidade de Contexto em sistemas sensíveis ao contexto.Propomos que métricas de Qualidade de Contexto sejam organizadas em níveis, reforçando a ideia de que, informações de contexto sejam qualificadas a partir de diferentes métricas baseadas tanto em aspectos dos sensores como em requisitos das aplicações. Uma métrica de primeiro nível representa a qualidade direta do contexto, podendo indicar a necessidade de este contexto ser novamente coletado ou de alterações na forma da coleta através dos sensores. Uma métrica de segundo nível caracteriza a qualidade de uma métrica de primeiro nível, podendo indicar a necessidade de atualização dos valores da métrica de primeiro nível, e em consequência, a necessidade de atualização do contexto. Uma métrica de QoC com baixa qualidade não estará qualificando adequadamente o contexto. Um valor baixo para Confidence, embora o período calculado para o Validity Time não tenha terminado e exista uma estimativa de que os veículos ainda se mantenham em comunicação, pode indicar a necessidade de que este valor seja novamente calculado, implicando também na atualização do contexto Communicating.

As métricas de QoC, Validity Time e Confidence podem ambas ser utilizadas pela própria rede veicular ou por aplicações veiculares para tomar decisões quanto ao uso das informações do contexto. Por exemplo, para a criação e a manutenção de rotas de comunicação. Ou mais ainda, para apoiar aplicações veiculares na tomada de decisão, em relação à execução de ações distribuídas com segurança.

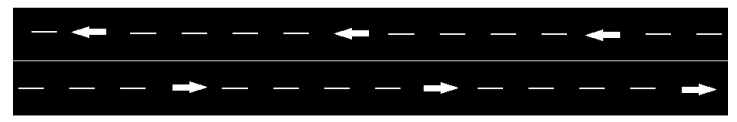

Figura 2: Estrada reta com vias em sentidos opostos

\section{AnÁlise da Confidence em um CENÁRIO DE SimulaçÃo}

O objetivo dessa análise é observar o comportamento do cálculo da métrica Confidence em relação aos aspectos de comunicação e mobilidade de um ambiente veicular. Para isto, adotamos a plataforma de software de simulação VEINS (Framework VeiNS - Vehicles in Network Simulation), a qual possibilita a implementação de cenários de tráfego, considerando aspectos de mobilidade e de comunicação concomitantemente. O Framework VEINS permite integrar o OMNeT++ (Objective Modular Network Testbed in $\mathrm{C}++$ ) para simulação do ambiente de comunicação veicular e o SUMO (Simulation of Urban Mobility) para a simulação do tráfego dos veículos em vias [18], [19], [16], [17].

Como a Confidence está relacionada às informações sobre as interações entre dois veículos, conjuntamente à dinâmica de uma VANET, consideramos indispensável realizar simulações exclusivamente com dois veículos, obtendo uma análise inicial criteriosa sobre o comportamento do cálculo proposto. Ter outros veículos nos cenários de simulação poderia criar tráfego nas vias, o que não traria impacto para o objetivo dessa análise, ou tráfego de comunicação podendo gerar falhas de comunicação.Por outro lado, injetamos falhas de comunicação em alguns experimentos através da configuração do ambiente de simulação, sem a necessidade de considerar mais veículos nestes experimentos.

Durante a trajetória os veículos se aproximam e se afastam entre si, até saírem do alcance de seus sinais de comunicação. 
Os experimentos foram realizados em cenários específicos e controlados, para avaliar a métrica Confidence, qualificando a confiança na Validity Time da comunicação entre os veículos.

No cenário utilizamos um mapa com uma estrada reta, em espaço livre e sem obstáculos (edificações, relevos etc.), que tem $2265 m$ de comprimento, possui duas vias e cada via possui duas faixas, como mostra a Figura 2. As setas na Figura 2 correspondem à direção dos veículos nas vias. Os veículos trafegam em direções opostas na mesma estrada. Usamos o modelo de mobilidade baseado em [20], provido pelo SUMO, que evita colisões entre veículos ao percorrer uma trajetória. A velocidade, aceleração e desaceleração de cada veículo podem variar, dentro de valores máximos configurados. Adotamos os seguintes valores: $\max S p e e d=13.89 \mathrm{~m} / \mathrm{s}$ para a velocidade, accel $=2.6 \mathrm{~m} / \mathrm{s}^{2}$ para a aceleração e decel $=4.5 \mathrm{~m} / \mathrm{s}^{2}$ para a desaceleração de cada veículo. Configuramos a velocidade máxima possível de um veículo como sendo igual a $V \max =41.67 \mathrm{~m} / \mathrm{s}$, tomando como referência a capacidade do motor de um carro popular.

No cenário, a comunicação entre os veículos utiliza o protocolo IEEE 802.11p disponibilizado pelo VeiNS. A potência do sinal de comunicação dos veículos é $t x$ Power $=20 \mathrm{~mW}$ e a sensibilidade para a captação do sinal de comunicação é igual a minPower Level $=-89 \mathrm{dBm}$. A distância do alcance do sinal de comunicação de cada veículo foi fixada em $250 \mathrm{~m}$, valor definido com base nos trabalhos propostos em [21] [22].

Em VANETs, perdas de mensagens impactam na atualização do contexto [23]. Assim, nas simulações adotamos cenários sem e com perda de mensagens de $25 \%$. Esta taxa de perda de mensagens considera os resultados encontrados em [23], com taxa de perdas de até $20 \%$, e em [15], com taxa de perdas de até 25\%. Além disto, utilizamos os períodos fixos e aleatórios de disseminação das mensagens. Para os períodos fixos usamos $0.5 s$ e $2 s$. Os períodos aleatórios variaram no intervalo $P=[0.5 s, 2 s]-$ tomando como base o trabalho apresentado em [21].

$\mathrm{Na}$ próxima seção apresentamos a análise do comportamento da métrica Confidence em relação ao cenário adotado.

\section{A. Análise dos Resultados Encontrados}

Como cada veículo está em sentido oposto, em uma via sem obstáculos e sem interferência de outros veículos, em cada execução a velocidade dos veículos manteve-se em aproximadamente $\approx 13.89 \mathrm{~m} / \mathrm{s}$ e a velocidade relativa em $\approx 27.78 \mathrm{~m} / \mathrm{s}$ ao longo da trajetória. Nestas condições, cada veículo permaneceu no alcance de comunicação do outro durante $18 \mathrm{~s}$. A duração de toda a simulação é de $125 \mathrm{~s}$. No instante de tempo $0 s$ os veículos começam a se aproximar, mas só no instante de $75 \mathrm{~s}$ aproximadamente, cada veículo entra no alcance de comunicação do outro e, consequentemente, começam a receber mensagens e atualizar os seus respectivos contextos de comunicação. No instante de $\approx 84 s$, os veículos começam a se afastar entre si e, no instante $93.2 \mathrm{~s}$, os veículos não conseguem mais trocar mensagens.

$\mathrm{Na}$ Figura 3 apresentamos resultados das simulações sem perda de mensagens. Os Gráficos 3a, 3c e 3e mostram o com- portamento de $\alpha_{r l}$ (Confidence) nos cenários com períodos de disseminação de mensagens de contexto com $0.5 \mathrm{~s}, 2 \mathrm{~s}$ e $P=[0 . s$ variável, respectivamente. Os gráficos $3 \mathrm{~b}, 3 \mathrm{~d}$ e $3 \mathrm{f}$ relacionam os valores das métricas age $r l, V T_{r l}$ e $V T_{r l}^{\text {min }}$ nos mesmos experimentos.

Nos gráficos 3b, 3d e 3f é possível observar o comportamento do $V T_{r l}$, sendo reduzido ao longo do tempo e tendendo a $0 s$, quando os veículos de fato se afastam e perdem sua

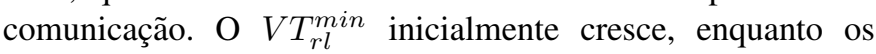
veículos se aproximam, e depois reduz tendendo a $0 s$, após os veículos cruzarem e começarem a se afastar entre si.

Entre os instantes $75 \mathrm{~s}$ e $\approx 78 \mathrm{~s}$, observa-se nos Gráficos $3 \mathrm{a}, 3 \mathrm{c}$ e $3 \mathrm{e}$ que os veículos estão se aproximando e entrando no alcance de comunicação do outro. Neste momento, $\alpha_{r l}$ é atenuado, pois $V T_{\min }$ ainda está muito pequeno em relação a $a g e_{r l}$. Isso significa que, apesar de estarem se aproximando, o $V T_{r l}$ ainda não está confiável, pois está próximo dos limites do alcance do sinal de comunicação.

No gráfico $3 \mathrm{~b}$, com um valor de $0.5 \mathrm{~s}$ no período de disseminação de mensagens de contexto, o cálculo de Age se mantém pequeno, em geral inferior a $V T_{r l}^{\text {min }}$, caracterizando poucas alterações no valor de Confidence, como visto no gráfico 3a. Apenas no final do experimento, quando os valores de $V T_{r l}$ e $V T_{r l}^{\text {min }}$ tendem a $0 s$, é que o valor de $\alpha_{r l}$ cai rapidamente, chegando a 0 .

No gráfico $3 \mathrm{~d}$, com um período de disseminação de mensagens igual a $2 s, a g e_{r l}$ tende a alcançar valores maiores do que o $V T_{r l}^{\min } \operatorname{logo}$ antes de cada atualização do contexto, e em consequência, $\alpha_{r l}$ (no gráfico 3c) sofre maiores variações, embora se mantendo próximo a 1 , uma vez que os valores de $V T_{r l}$ durante boa parte do experimento são altos em relação aos valores do $V T_{r l}^{\text {min }}$.

Nos gráficos $3 \mathrm{e}$ e $3 \mathrm{f}$, podemos perceber que, o fato de o período de disseminação de mensagens ser variável no intervalo dos valores adotados, não gera impactos no cálculo das métricas, as quais representam adequadamente a situação do contexto.

Os gráficos da Figura 4 apresentam as mesmas situações dos gráficos da Figura 3, mas considerando perda de mensagens. $\mathrm{O}$ efeito da perda de mensagens gerou valores maiores para a métrica $a g e_{r l}$, e consequentemente, maiores variações em $\alpha_{r l}$. Estas variações são verificáveis, em especial no gráfico $4 \mathrm{c}$ no momento $80 \mathrm{~s}$ e também entre $82 \mathrm{~s}$ e $84 \mathrm{~s}$, do experimento. Também nos gráficos $4 \mathrm{a}$ e $4 \mathrm{e}$ é possível observar estas variações no valor de $\alpha_{r l}$. Nestes casos, estas variações podem ser utilizadas como indicativos de falhas, como a perda de mensagens, podendo ser utilizados nas decisões pelo envio imediato de novas mensagens de contexto, por exemplo.

Em todos os gráficos observamos que o valor de $\alpha_{r l}$ se mantém próximo a 1 durante o maior tempo dos experimentos, só tendendo a 0 quando os experimentos se aproximam do final, com os veículos de fato distantes e se movimentando para fora do alcance de seus sinais de comunicação. Nestas situações, os valores de $V T_{r l}$ e $V T_{r l}^{\text {min }}$ se tornam muito pequenos, tendendo a $0 s$, e portanto, inferiores ao valor de age $r$. 


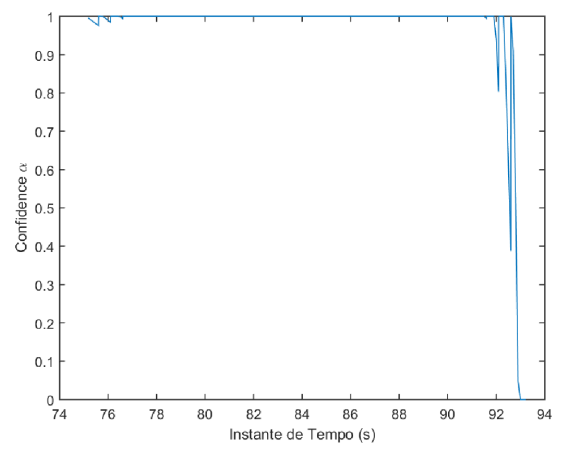

(a) $\alpha(0.5 s)$

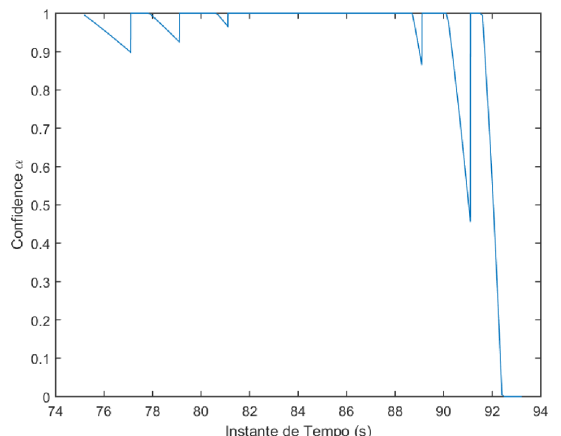

(c) $\alpha(2 s)$

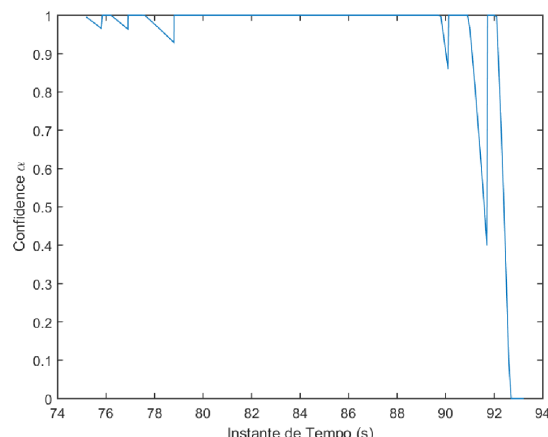

(e) $\alpha(P s)-P=[0.5 s, 2 s]$

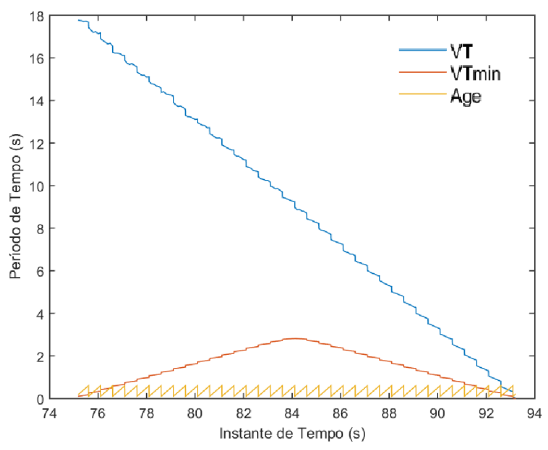

(b) $a g e_{r l}(0.5 s), V T_{r l}(0.5 s)$ e $V T_{r l}^{\min }(0.5 s)$

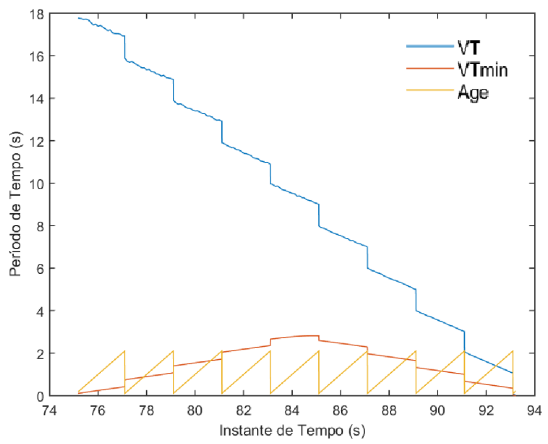

(d) $a g e_{r l}(2 s), V T_{r l}(2 s)$ e $V T_{r l}^{\min }(2 s)$

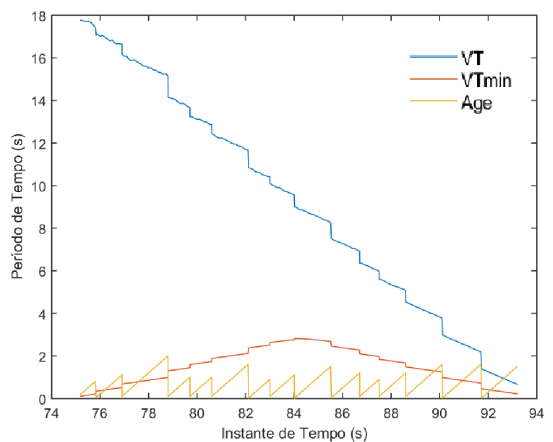

(f) $a g e_{r l}(P s), V T_{r l}(P s)$ e $V T_{r l}^{\min }(P s)-P=$ $[0.5 s, 2 s]$

Figura 3: Resultados em cenários sem perda de mensagens

\section{Trabalhos Relacionados}

Diversos trabalhos usam o conceito de contexto em VANETs, desenvolvendo redes veiculares cientes do contexto [24], [6], [25]. Estes trabalhos usam informações coletadas da rede veicular, tais como posição, direção e sentido do movimento, velocidade, estado do tráfego, obstáculos e características da comunicação sem fio, incluindo hardware e software.

Sepulcre e Gozalvez [8] apresentam uma arquitetura para redes veiculares heterogêneas cientes do contexto. Assumem um contexto de tráfego de veículos e sua mobilidade. A arquitetura define os componentes responsáveis pela aquisição e armazenamento do contexto, pela gestão de perfis de comunicação baseados no contexto, e também pela decisão sobre a adaptação da configuração de aplicações, a partir do contexto.

Vahdat-Nejad et al [7] analisa diversos projetos de aplicações veiculares cientes do contexto. $\mathrm{O}$ trabalho propõe um framework para a classificação das aplicações, dividido em 3 dimensões: ambiente, sistema e aplicação e ciência de contexto. O framework é utilizado para a revisão de diversas aplicações de transportes veiculares inteligentes (ITS), a partir das 3 dimensões definidas.

Ghaleb et al [5] apresentam um serviço de detecção de comportamento inadequado no fornecimento de informações de contexto de mobilidade, por parte de um veículo, por conta 


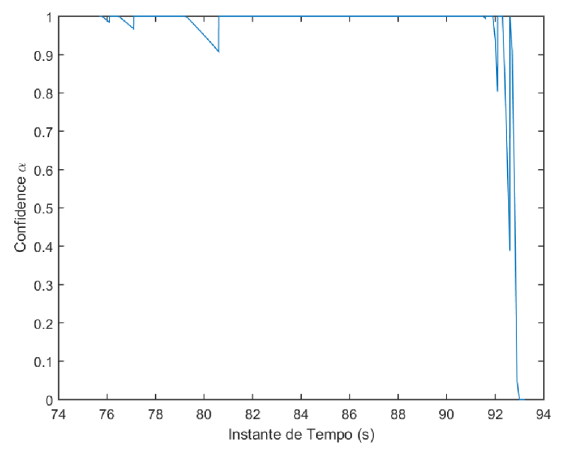

(a) $\alpha(0.5 s)$

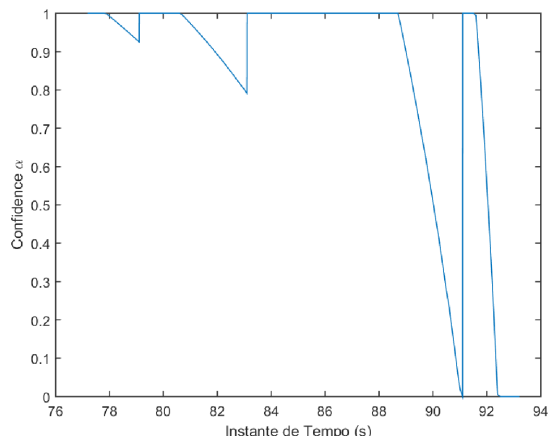

(c) $\alpha(2 s)$

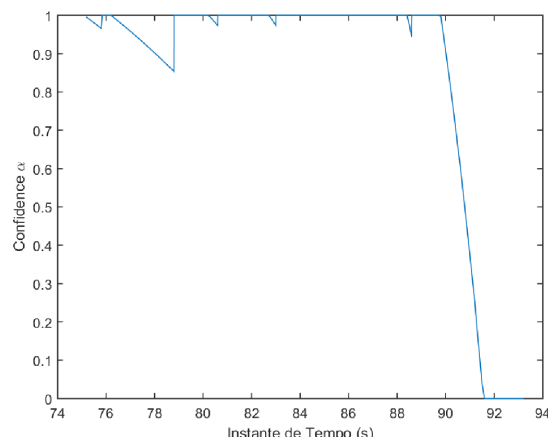

(e) $\alpha(P s)-P=[0.5 s, 2 s]$

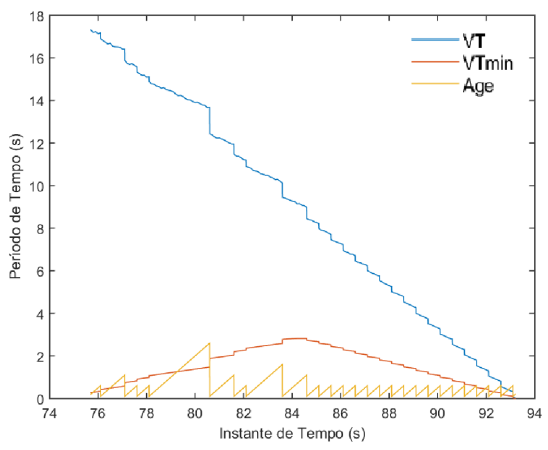

(b) $a g e_{r l}, V T_{r l}$ e $V T_{r l}^{\text {min }}$

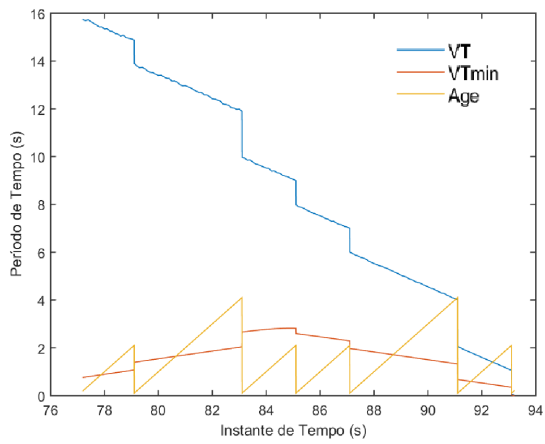

(d) $a g e_{r l}(2 s), V T_{r l}(2 s)$ e $V T_{r l}^{\min }(2 s)$

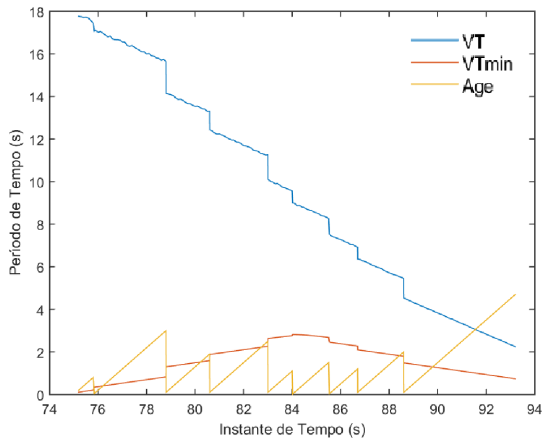

(f) $a g e_{r l}(P s), V T_{r l}(p s)$ e $V T_{r l}^{\min }(P s)-P=$ $[0.5 s, 2 s]$

Figura 4: Resultados em cenários com perdas de mensagens

de ataques. Considera-se que em aplicações veiculares críticas, como prevenção de colisões, informações incorretas podem dificultar as decisões dos veículos.

Guo et al [26] propõem um modelo ciente de contexto para avaliar a confiabilidade de mensagens a serem utilizadas pelo condutor, para evitar que mensagens falsas influenciem suas decisões. O sistema também utiliza um modelo de aprendizagem por reforço. Diferente do nosso trabalho, estes não lidam com à duração da utilidade da informação em relação aos aspectos de comunicação veicular.

Por outro lado, pouco tem sido apresentado com relação ao uso das métricas de Qualidade do Contexto em redes veiculares. Yasar et al [27] definem métricas de QoC rela- tivas às mensagens que estão sendo trocadas pelas aplicações veiculares. Foram propostos parâmetros relacionados com as mensagens sendo trocadas, tais como: relevância temporal das mensagens, completude da informação, prioridade da informação e relevância espacial da informação. Estas métricas são utilizadas em conjunto com um conceito de reputação, para decidir acerca da prioridade a ser fornecida ao processamento de cada mensagem.

Diferente de [27], propomos métricas de QoC aplicadas ao contexto de mobilidade e comunicação, que podem ser utilizadas pela própria rede veicular em decisões sobre o encaminhamento de mensagens, e pelas aplicações sobre a segurança em executar seus diversos processamentos. 


\section{CONCLUSÃO}

Neste artigo analisamos e discutimos a métrica de Qualidade de Contexto Confidence, mostrando o seu comportamento, e como ela qualifica os valores calculados para o Validity Time. Estas métricas de QoC foram apresentadas originalmente em [14], juntamente com um contexto de mobilidade e comunicação para redes veiculares. Também discutimos o conceito de Qualidade de Contexto em níveis, conceito que apresentamos neste artigo, mostrando Confidence como uma métrica de QoC que qualifica uma outra métrica de QoC, no caso a Validity Time.

Nesta análise mostramos como a métrica Confidence pode ser utilizada como um balizador para indicar um período adequado para a monitoração do contexto, ou situações nas quais ações precisem ser adotadas para atualizar informações, e desta forma ter parâmetros para auxiliar as decisões do serviço de comunicação. Através desta métrica definimos um grau de confiança no valor de Validity Time relacionando situações desejáveis (informações recentes do contexto) e indesejáveis (contexto sem atualizações). Com o cálculo proposto é possível definir novas condições do ambiente e incorporar novos graus de confiança intermediários entre $V T_{r l}$ e $V T_{r l}^{\min }$, a partir de requisitos apresentados pelas aplicações veiculares.

Por fim, analisamos Confidence por meio de resultados obtidos em ambiente simulado, com o Framework VeiNS. Os resultados apresentam um comportamento consistente de Confidence, qualificando a confiabilidade do valor de Validity Time relativo à idade do contexto utilizado no cálculo desta métrica (Age). Quanto mais antigo for o contexto, menor a confiança no Validity Time. Os experimentos também mostram que falhas na comunicação gerando maior variação na idade do contexto são identificadas por Confidence. Com tais resultados, em trabalhos futuros, analisaremos Confidence em cenários com mais veículos, vias diferentes, entre outros.

Embora o conceito de Contexto esteja sendo investigado em redes veiculares, muito pouco tem sido proposto com relação a Qualidade de Contexto. Neste sentido, nosso trabalho vem corrigir esta falha, propondo formas de prover informações seguras e confiáveis para as redes e suas aplicações.

\section{REFERÊNCIAS}

[1] B. N. Schilit and M. M. Theimer, "Disseminating active map information to mobile hosts," IEEE Network, vol. 8, no. 5, pp. 22-32, 1994.

[2] M. Baldauf, S. Dustdar, and F. Rosenberg, "A survey on context-aware systems," International Journal of Ad Hoc and Ubiquitous Computing, vol. 2, no. 4, pp. 263-277, 2007.

[3] G. D. Abowd, A. K. Dey, P. J. Brown, N. Davies, M. Smith, and P. Steggles, "Towards a better understanding of context and contextawareness," in Handheld and ubiquitous computing. Springer, 1999, pp. 304-307.

[4] S. Pajares Ferrando and E. Onaindia, "Context-aware multi-agent planning in intelligent environments," Information Sciences, vol. 227, pp. 22-42, 2013.

[5] F. A. Ghaleb, M. A. Maarof, A. Zainal, B. A. S. Al-rimy, F. Saeed, and T. Al-Hadhrami, "Hybrid and multifaceted context-aware misbehavior detection model for vehicular ad hoc network," IEEE Access, vol. 7, pp. 159 119-159 140, 2019.

[6] F. Dressler, F. Klingler, C. Sommer, and R. Cohen, "Not all vanet broadcasts are the same: Context-aware class based broadcast," IEEE/ACM Transactions on Networking, 2018.
[7] H. Vahdat-Nejad, A. Ramazani, T. Mohammadi, and W. Mansoor, "A survey on context-aware vehicular network applications," Vehicular Communications, vol. 3, pp. 43-57, 2016.

[8] M. Sepulcre and J. Gozalvez, "Context-aware heterogeneous v2x communications for connected vehicles," Computer Networks, vol. 136, pp. 13-21, 2018.

[9] T. Buchholz, A. Küpper, and M. Schiffers, "Quality of context: What it is and why we need it," in Proceedings of the workshop of the HP OpenView University Association, vol. 2003, 2003.

[10] A. Manzoor, H.-L. Truong, and S. Dustdar, "Using quality of context to resolve conflicts in context-aware systems," in Quality of Context. Springer, 2009, pp. 144-155.

[11] -Quality of context: models and applications for context-aware systems in pervasive environments," The Knowledge Engineering Review, vol. 29, no. 02, pp. 154-170, 2014.

[12] P. Bellavista, A. Corradi, M. Fanelli, and L. Foschini, "A survey of context data distribution for mobile ubiquitous systems," ACM Comput. Surv., vol. 44, no. 4, pp. 24:1-24:45, Sep. 2012. [Online]. Available: http://doi.acm.org/10.1145/2333112.2333119

[13] N. Agoulmine et al., "A quality-aware approach for resolving context conflicts in context-aware systems," in Embedded and Ubiquitous Computing (EUC), 2011 IFIP 9th International Conference on. IEEE, 2011, pp. 229-236.

[14] M. Sá and S. Gorender, "Quality of context for vanets: Qoc metrics for connectivity in vanets." in 2019 18th International Conference on Ad-Hoc Networks and Wireless (ADHOC-NOW). Springer, 2019, pp. $420-431$.

[15] — "Monitoração inteligente de estados de comunicação para aplicações sobre redes veiculares," in IX Encontro Nacional de Inteligência Artificial, ser. ENIA'2012, Paraná, Brasil, October 2012.

[16] A. Wegener, M. Piórkowski, M. Raya, H. Hellbrück, S. Fischer, and J. Hubaux, "Traci: an interface for coupling road traffic and network simulators," in Proceedings of the 11th communications and networking simulation symposium. ACM, 2008, pp. 155-163.

[17] C. Sommer, R. German, and F. Dressler, "Bidirectionally coupled network and road traffic simulation for improved ivc analysis," Mobile Computing, IEEE Transactions on, vol. 10, no. 1, pp. 3-15, 2011.

[18] D. Krajzewicz, G. Hertkorn, C. Rossel, and P. Wagner, "Sumo (simulation of urban mobility)," Proceedings of the 4th Middle East Symposium on Simulation and Modelling (MESM20002), pp. 183-187, 2002.

[19] A. Varga et al., "The omnet++ discrete event simulation system," in Proceedings of the European Simulation Multiconference (ESM2001), 2001, pp. 319-324.

[20] S. Krauß, "Microscopic modeling of traffic flow: Investigation of collision free vehicle dynamics," Ph.D. dissertation, DLR Deutsches Zentrum fuer Luft-und Raumfahrt eV, Koeln (Germany). Abt. Unternehmensorganisation und-informatio, 1998.

[21] P. Shankar, T. Nadeem, J. Rosca, and L. Iftode, "Cars: Context-aware rate selection for vehicular networks," in Network Protocols, 2008. ICNP 2008. IEEE International Conference on. IEEE, 2008, pp. 1-12.

[22] J. Ott and D. Kutscher, "Drive-thru internet: Ieee 802.11 b for"automobile" users," in IEEE INFOCOM 2004, vol. 1. IEEE, 2004.

[23] F. Lyu, N. Cheng, H. Zhu, H. Zhou, W. Xu, M. Li, and X. S. Shen, "Intelligent context-aware communication paradigm design for iovs based on data analytics," IEEE Network, vol. 32, no. 6, pp. 74-82, 2018.

[24] K. Paridel, T. Mantadelis, D. Preuveneers, G. Janssens, Y. Vanrompay, Y. Berbers et al., "Analyzing the efficiency of context-based grouping on collaboration in vanets with large-scale simulation," Journal of Ambient Intelligence and Humanized Computing, vol. 5, no. 4, pp. 475-490, 2014.

[25] V. Alagar and K. Wan, "Context-aware trust-based management of vehicular ad-hoc networks (vanets)," in 2015 IEEE 12th Intl Conf on Ubiquitous Intelligence and Computing and 2015 IEEE 12th Intl Conf on Autonomic and Trusted Computing and 2015 IEEE 15th Intl Conf on Scalable Computing and Communications and Its Associated Workshops (UIC-ATC-ScalCom). IEEE, 2015.

[26] J. Guo, X. Li, Z. Liu, J. Ma, C. Yang, J. Zhang, and D. Wu, "Trove: A context-awareness trust model for vanets using reinforcement learning," IEEE INTERNET OF THINGS, vol. 7, no. 7, pp. 6647-6662, july 2020.

[27] A. Yasar, K. Paridel, D. Preuveneers, and Y. Berbers, "When efficiency matters: towards quality of context-aware peers for adaptive communication in vanets," in Intelligent Vehicles Symposium (IV), 2011 IEEE. IEEE, 2011, pp. 1006-1012. 